\title{
SHARP COUNTEREXAMPLES FOR STRICHARTZ ESTIMATES FOR LOW REGULARITY METRICS
}

\author{
Hart F. Smith and Daniel Tataru
}

\section{Introduction}

In this paper we produce examples of time independent $C^{s}$ metrics, for $0 \leq$ $s \leq 2$, and solutions $u$ to the wave equation for such metrics, which establish sharp lower bounds on the index of the Sobolev norm of the initial data of $u$ required to bound mixed $L^{p} L^{q}$ norms of $u$.

Consider a second order hyperbolic operator on $[0,1] \times \mathbb{R}^{n}$,

$$
P\left(t, x, \partial_{t}, \partial_{x}\right)=\partial_{t}^{2}-\partial_{x_{i}} g^{i j}(t, x) \partial_{x_{j}}
$$

and the following estimates of Strichartz type

$$
\begin{aligned}
\|u\|_{L_{t}^{p} L_{x}^{q}\left([0,1] \times \mathbb{R}^{n}\right)} \leq C\left(\|u\|_{L_{t}^{\infty}\left([0,1] ; H_{x}^{\gamma}\left(\mathbb{R}^{n}\right)\right)}+\right. & \left\|\partial_{t} u\right\|_{L_{t}^{\infty}\left([0,1] ; H_{x}^{\gamma-1}\left(\mathbb{R}^{n}\right)\right)} \\
& \left.+\|P u\|_{L_{t}^{1}\left([0,1] ; H_{x}^{\gamma-1}\left(\mathbb{R}^{n}\right)\right)}\right) .
\end{aligned}
$$

If the coefficients of $P$ are smooth, then it is known that these estimates hold for $(p, q)$ satisfying

$$
\frac{1}{p}=\left(\frac{n-1}{2}\right)\left(\frac{1}{2}-\frac{1}{q}\right), \quad 2 \leq q \leq \frac{2(n-1)}{n-3},
$$

provided $(n, p, q) \neq(3,2, \infty)$, where the Sobolev index $\gamma$ is given by

$$
\gamma=\left(\frac{n+1}{2}\right)\left(\frac{1}{2}-\frac{1}{q}\right)
$$

On the other hand, in [3] there were constructed for each $s<2$ examples of $P$ with time independent coefficients of regularity $C^{s}$ for which the same estimates fail to hold. The first author then showed in [1] that, in space dimensions 2 and 3 , the estimates do hold if the coefficients of $P$ are $C^{1,1}$. The second author subsequently showed in [4] that the estimates hold for $C^{2}$ metrics in all space dimensions, and that for operators with $C^{s}$ coefficients, $0<s<2$, such estimates hold provided that $\gamma$ is replaced by $\gamma+\sigma / p$, where $\sigma=\frac{2-s}{2+s}$. Indeed, [5] showed that such estimates hold under the condition that $s$ derivatives of the coefficients belong to $L_{t}^{1} L_{x}^{\infty}$, which is important for applications to quasilinear wave equations.

Received October 23, 2001.

This research was supported in part by National Science Foundation grants DMS-9970407 and DMS-9622942. 
The counterexamples of [3] do not coincide with the estimates established by [4], however. In this paper we remedy this gap, by producing examples of time independent $C^{s}$ metrics, with $0 \leq s \leq 2$, which show that the results established in [4] are indeed best possible.

Theorem 1. Let $0 \leq s \leq 2$, and suppose that $(p, q)$ satisfy (2). Assume that the estimate (1) holds with a constant $C$ depending only on the $C^{s}$ norm of the coefficients, for all metrics $g^{i j}(x)$ sufficiently close in the uniform norm to the Euclidean metric $\delta^{i j}$. Then

$$
\gamma \geq\left(\frac{n+1}{2}\right)\left(\frac{1}{2}-\frac{1}{q}\right)+\frac{\sigma}{p}, \quad \sigma=\frac{2-s}{2+s} .
$$

We remark that this construction also produces examples of $C^{s}$ metrics, $1 \leq$ $s \leq 2$, which show that the closely related spectral projection estimates for $C^{s}$ metrics established by the first author [2] are best possible. For the spectral projection estimates, however, the counterexamples of [3] were already sharp.

\section{An explicit example}

Here we give a simple explicit construction, but which works only for $s \geq \frac{2}{3}$. In the next section we explain how to modify this construction in order to make it work for $0 \leq s<\frac{2}{3}$. We work with variables $t \in \mathbb{R}, x \in \mathbb{R}, y \in \mathbb{R}^{n-1}$. For $\xi>0$ a real number, we let

$$
\begin{gathered}
u_{\xi}(t, x, y)=e^{i \xi(t-x)-i t\left(\frac{n-1}{2}\right)-\frac{1}{2} \xi|y|^{2}}, \\
P\left(y, \partial_{t}, \partial_{x}, \partial_{y}\right)=\left(\partial_{t}^{2}-\left(1+|y|^{2}\right) \partial_{x}^{2}-\Delta_{y}\right),
\end{gathered}
$$

and observe that

$$
P u_{\xi}(t, x, y)=-\left(\frac{n-1}{2}\right)^{2} u_{\xi}(t, x, y)
$$

For an index $0 \leq s \leq 2$ we set

$$
\delta=\frac{2}{2+s}, \quad \sigma=\frac{2-s}{2+s},
$$

and note that $2 \delta=1+\sigma$.

We now fix $s$, and make a change of variables by scaling $(t, x, y)$ by $\lambda^{\sigma}$, and replacing $\xi$ by $r \lambda^{1-\sigma}$, to obtain

$$
u_{r}^{\lambda}(t, x, y)=e^{i r \lambda(t-x)-i \lambda^{\sigma} t\left(\frac{n-1}{2}\right)-\frac{1}{2} r \lambda^{2 \delta}|y|^{2}},
$$

which satisfies

$$
P_{\lambda} u_{r}^{\lambda}(t, x, y)=-\left(\frac{n-1}{2}\right)^{2} \lambda^{2 \sigma} u_{r}^{\lambda}(t, x, y)
$$

where

$$
P_{\lambda}\left(y, \partial_{t}, \partial_{x}, \partial_{y}\right)=P\left(\lambda^{\sigma} y, \partial_{t}, \partial_{x}, \partial_{y}\right)=\left(\partial_{t}^{2}-\left(1+\lambda^{2 \sigma}|y|^{2}\right) \partial_{x}^{2}-\Delta_{y}\right) .
$$


We next fix a smooth, nonnegative bump function $\beta$ supported in the interval $[1,2]$, and set

$$
u^{\lambda}(t, x, y)=\frac{1}{(\log \lambda)^{2}} \int \beta\left((\log \lambda)^{-2} r\right) u_{r}^{\lambda}(t, x, y) d r .
$$

The function $u^{\lambda}$ is essentially a smooth bump function of size 1 localized to the set $|x-t| \leq \lambda^{-1}(\log \lambda)^{-2},|y| \leq \lambda^{-\delta}(\log \lambda)^{-1}$. Rather than obtain pointwise estimates, though, it is easier to work with weighted $L^{2}$ estimates. Thus, we note the following two inequalities:

$$
\begin{gathered}
\int\left|u^{\lambda}(t, x, y)\right|^{2} d x d y \approx \lambda^{-1-(n-1) \delta}(\log \lambda)^{-n-1} \\
\int\left(1+\lambda^{2}(\log \lambda)^{4}|t-x|^{2}+\lambda^{2 \delta}(\log \lambda)^{2}|y|^{2}\right)^{n}\left|u^{\lambda}(t, x, y)\right|^{2} d x d y \\
\leq C \lambda^{-1-(n-1) \delta}(\log \lambda)^{-n-1}
\end{gathered}
$$

The first follows by the Plancherel theorem applied to the $x$ variable. The second follows by noting that

$$
\begin{aligned}
& \lambda^{j}(\log \lambda)^{2 j}(t-x)^{j} u^{\lambda}(t, x, y) \\
& \quad=e^{-i \lambda^{\sigma} t\left(\frac{n-1}{2}\right)} \int e^{i r \lambda(t-x)}(\log \lambda)^{2 j} \partial_{r}^{j}\left(e^{-\frac{1}{2} r \lambda^{2 \delta}|y|^{2}} \beta\left((\log \lambda)^{-2} r\right)\right) d r,
\end{aligned}
$$

and applying Plancherel as before.

Together, (3) and (4) and the Schwarz inequality imply

$$
\begin{aligned}
& \int\left(1+\lambda^{2}(\log \lambda)^{4}|t-x|^{2}+\lambda^{2 \delta}(\log \lambda)^{2}|y|^{2}\right)^{-n}\left|u^{\lambda}(t, x, y)\right|^{2} d x d y \\
& \approx \lambda^{-1-(n-1) \delta}(\log \lambda)^{-n-1}
\end{aligned}
$$

which by Holder's inequality implies that, for $2 \leq q \leq \infty$,

$$
\left\|u^{\lambda}(t, \cdot)\right\|_{L^{q}\left(\mathbb{R}^{n}\right)} \geq c \lambda^{-(1+(n-1) \delta) / q}(\log \lambda)^{-(n+1) / q},
$$

exactly the bounds for a suitably localized bump function of size 1. On the other hand, it is easy to compute the Sobolev space bounds

$$
\begin{aligned}
& \left\|u_{t}^{\lambda}(t, \cdot)\right\|_{H^{\gamma-1}\left(\mathbb{R}^{n}\right)}+\left\|u^{\lambda}(t, \cdot)\right\|_{H^{\gamma}\left(\mathbb{R}^{n}\right)} \\
& \leq C \lambda^{\gamma-(1+(n-1) \delta) / 2}(\log \lambda)^{2 \gamma-(n+1) / 2} \\
& \left\|P_{\lambda} u^{\lambda}(t, \cdot)\right\|_{H^{\gamma-1}\left(\mathbb{R}^{n}\right)} \leq C \lambda^{\gamma-1+2 \sigma-(1+(n-1) \delta) / 2}(\log \lambda)^{2(\gamma-1)-(n+1) / 2}
\end{aligned}
$$


If the Strichartz estimate (1) holds uniformly for $P_{\lambda}$ and $u_{\lambda}$, then by (5), (6) and (7) we must have

(8) $\lambda^{-\frac{1+(n-1) \delta}{q}}(\log \lambda)^{-\frac{n+1}{q}}$

$$
\leq C \lambda^{\gamma-\frac{1+(n-1) \delta}{2}}(\log \lambda)^{2 \gamma-\frac{n+1}{2}}\left(1+(\log \lambda)^{-2} \lambda^{2 \sigma-1}\right) .
$$

If $s \geq 2 / 3$, then $2 \sigma \leq 1$, and therefore we must have

$$
\gamma \geq\left(\frac{1}{2}-\frac{1}{q}\right)(1+(n-1) \delta)
$$

which compared to the smooth case involves a loss of derivatives of the following degree

$$
\left(1+(n-1) \delta-\frac{n+1}{2}\right)\left(\frac{1}{2}-\frac{1}{q}\right)=(2 \delta-1) \frac{1}{p}=\frac{\sigma}{p} .
$$

This would conclude the proof of Theorem 1 if the coefficients of the operators $P_{\lambda}$ were uniformly bounded in $C^{s}$. While this is not true, the bound

$$
\int_{|y| \geq \lambda^{-\delta}}\left|\partial_{t, x, y}^{\alpha} u^{\lambda}(t, x, y)\right|^{2} d x d y \leq C_{N, \alpha} \lambda^{-N}
$$

shows that we can freely modify the coefficients of $P_{\lambda}$ outside the ball $\{|y| \leq$ $\left.\lambda^{-\delta}\right\}$. Thus, let $a(y)$ denote a positive smooth function, such that

$$
a(y)=|y|^{2} \quad \text { if } \quad|y| \leq 1, \quad a(y)=0 \quad \text { if } \quad|y| \geq 2,
$$

and set

$$
P_{\lambda}^{1}=\partial_{t}^{2}-\left(1+\lambda^{2 \sigma-2 \delta} a\left(\lambda^{\delta} y\right)\right) \partial_{x}^{2}-\Delta_{y}
$$

Note that

$$
\left.\left.\| \lambda^{2 \sigma-2 \delta} a\left(\lambda^{\delta} y\right)\right)\left\|_{C^{0}} \leq C \lambda^{2 \sigma-2 \delta}, \quad\right\| \lambda^{2 \sigma-2 \delta} a\left(\lambda^{\delta} y\right)\right) \|_{C^{2}} \leq C \lambda^{2 \sigma} .
$$

Since $(2 \sigma-2 \delta)(2-s)+2 \sigma s=0$, it follows that $P_{\lambda}^{1}$ has $C^{s}$ coefficients, uniformly over $\lambda$. Furthermore, the coefficients of $P_{\lambda}^{1}$ converge in the $L^{\infty}$ norm to those of the usual d'Alembertian $\partial_{t}^{2}-\partial_{x}^{2}-\Delta_{y}$ as $\lambda \rightarrow \infty$.

\section{A modified example}

The reason that the previous example fails for $s<2 / 3$ is that $P_{\lambda} u_{r}^{\lambda}$ is too large. To remedy this, we seek modified functions $u_{\xi}$ and operators $P$ of the form

$$
\begin{gathered}
u_{\xi}(t, x, y)=a(y) e^{i \xi(t-x)+i \alpha t-\xi \phi(y)}, \\
P\left(y, \partial_{t}, \partial_{x}, \partial_{y}\right)=\partial_{t}^{2}-g(y) \partial_{x}^{2}-\Delta_{y},
\end{gathered}
$$

with $a, g, \phi$ smooth on some ball about 0 , spherically symmetric, and with $a(0)=g(0)=1, \Delta \phi(0)>0, \alpha>0$, and

$$
P u_{\xi}=0 \text {. }
$$


Given such a function $u_{\xi}$ and an operator $P$ then we can substitute them in the argument of the previous section, and so obtain the desired counterexamples in the full range $0 \leq s \leq 2$.

We compute

$$
P u_{\xi}=\left(-(\xi+\alpha)^{2}+\xi^{2} g(y)-\xi^{2}|\nabla \phi|^{2}+\xi \Delta \phi-\frac{\Delta a}{a}+2 \xi \frac{\nabla a \cdot \nabla \phi}{a}\right) u_{\xi} .
$$

By requiring that this vanish for all $\xi$ we obtain the following nonlinear system for $a, g, \phi$ :

$$
\left\{\begin{array}{l}
\Delta a+\alpha^{2} a=0 \\
\Delta \phi+2 \frac{\nabla a \cdot \nabla \phi}{a}-2 \alpha=0 \\
g=1+|\nabla \phi|^{2}
\end{array}\right.
$$

The first equation permits an analytic, spherically symmetric solution,

$$
a(y)=c_{n} \int_{S^{n-2}} e^{i \alpha\langle y, \eta\rangle} d \sigma(\eta) .
$$

The function $g$ is uniquely determined by the third equation, so it remains to solve the second equation for $\phi$. Since $a$ has zeros, we only obtain a local solution $\phi$ for $y$ near 0 . If we express $\nabla a / a$ and $\phi$ as formal power series near 0 ,

$$
\frac{\nabla a(y)}{a(y)}=y \sum_{k=1}^{\infty} a_{2 k}|y|^{2 k-2}, \quad \phi(y)=\sum_{k=1}^{\infty} b_{2 k}|y|^{2 k},
$$

then we derive the recurrence relation

$$
k(2 k+n-3) b_{2 k}=-\sum_{j=1}^{k-1} 2 j a_{2(k-j)} b_{2 j}, \quad k \geq 2,
$$

with the initial condition

$$
b_{2}=\frac{\alpha}{n-1}>0
$$

This implies that

$$
\left|b_{2 k}\right|<\max _{1 \leq j \leq k-1}\left|a_{2(k-j)} b_{2 j}\right| .
$$

Since $a$ is analytic near 0 we have

$$
\left|a_{2 k}\right| \leq M^{k}
$$

where $M^{-1}$ is the distance to the first complex 0 of $a$. Combined with the previous inequality, this inductively leads to the bound

$$
\left|b_{2 k}\right| \leq M^{k-1} b_{2}
$$

which guarantees that the formal series for $b$ generates an analytic function near 0 . 
We remark that for dimension $n=2$ one can explicitly solve the above system to obtain

$$
a(y)=\cos \alpha y, \quad \phi(y)=y \tan \alpha y \text {. }
$$

\section{References}

[1] H.F. Smith, A parametrix construction for wave equations with $C^{1,1}$ coefficients, Ann. Inst. Fourier (Grenoble) 48 (1998) no. 3, 797-835.

[2] H. F. Smith, Eigenfunction estimates for low regularity metrics, preprint.

[3] H. F. Smith and C. D. Sogge, On Strichartz and eigenfunction estimates for low regularity metrics, Math. Res. Lett. 1 (1994), no. 6, 729-737.

[4] D. Tataru, Strichartz estimates for second order hyperbolic operators with nonsmooth coefficients. II, Amer. J. Math. 123 (2001), no. 3, 385-423.

[5] D. Tataru, Strichartz estimates for second order hyperbolic operators with nonsmooth coefficients. III, to appear in J. Amer. Math. Soc.

Department of Mathematics, University of Washington, Seattle, WA 98195, U.S.A.

E-mail address: hart@math.washington.edu

Department of Mathematics, University of California, Berkeley, CA 94720, U.S.A.

E-mail address: tataru@math.berkeley.edu 